\title{
Parthenogenetic chimaerism/mosaicism with a Silver-Russell syndrome-like phenotype
}

\author{
K Yamazawa, ${ }^{1,2}$ K Nakabayashi, ${ }^{3}$ M Kagami, ${ }^{1}$ T Sato, ${ }^{1}$ S Saitoh, ${ }^{4}$ R Horikawa, \\ N Hizuka, ${ }^{6}$ T Ogata $^{1}$
}

- Additional figures, tables and an appendix are published online only. To view these files, please visit the journal online (http://jmg.bmi.com).

${ }^{1}$ Departments of Endocrinology and Metabolism, National Research Institute for Child Health and Development, Tokyo, Japan

2Department of Physiology, Development \& Neuroscience, University of Cambridge, Cambridge, UK

${ }^{3}$ Maternal-Fetal Biology, National Research Institute for Child Health and Development,

Tokyo, Japan

${ }^{4}$ Department of Pediatrics, Hokkaido University Graduate School of Medicine, Sapporo, Japan

${ }^{5}$ Division of Endocrinology and Metabolism, National Children's Hospital, Tokyo, Japan ${ }^{6}$ Department of Medicine, Institute of Clinical Endocrinology, Tokyo Women's Medical University, Tokyo, Japan

\section{Correspondence to}

Dr Tsutomu Ogata, Department of Endocrinology and Metabolism, National Research Institute for Child Health and Development, 2-10-1 Ohkura, Setagaya, Tokyo 157-8535, Japan; tomogata@nch.go.jp

Received 20 March 2010 Revised 6 May 2010 Accepted 8 May 2010 Published Online First 3 August 2010

This paper is freely available online under the BMJ Journals unlocked scheme, see http:// jmg.bmj.com/site/about/ unlocked.xhtml

\section{ABSTRACT}

Introduction We report a 34-year-old Japanese female with a Silver-Russell syndrome (SRS)-like phenotype and a mosaic Turner syndrome karyotype $(45, X / 46, X X)$.

Methods/Results Molecular studies including methylation analysis of 17 differentially methylated regions (DMRs) on the autosomes and the XIST-DMR on the $X$ chromosome and genome-wide microsatellite analysis for 96 autosomal loci and $30 \mathrm{X}$ chromosomal loci revealed that the $46, X X$ cell lineage was accompanied by maternal uniparental isodisomy for all chromosomes (upid(AC)mat), whereas the $45, X$ cell lineage was associated with biparentally derived autosomes and a maternally derived $X$ chromosome. The frequency of the $46, X X$ upid $(A C)$ mat cells was calculated as $84 \%$ in leukocytes, $56 \%$ in salivary cells, and $18 \%$ in buccal epithelial cells.

Discussion The results imply that a parthenogenetic activation took place around the time of fertilisation of a sperm missing a sex chromosome, resulting in the generation of the upid(AC)mat $46, X X$ cell lineage by endoreplication of one blastomere containing a female pronucleus and the $45, \mathrm{X}$ cell lineage by union of male and female pronuclei. It is likely that the extent of overall (epi)genetic aberrations exceeded the threshold level for the development of SRS phenotype, but not for the occurrence of other imprinting disorders or recessive Mendelian disorders.

Although a mammal with maternal uniparental disomy for all chromosomes (upd(AC)mat) is incompatible with life because of genomic imprinting, ${ }^{1}$ a mammal with a upd(AC)mat cell lineage could be viable in the presence of a coexisting normal cell lineage. In the human, Strain et $a l^{2}$ have reported 46,XX peripheral blood cells with maternal uniparental isodisomy for all chromosomes (upid(AC)mat) in a 1.2-year-old phenotypically male patient with aggressive behaviour, hemifacial hypoplasia and normal birth weight. Because of the 46,XX disorders of sex development, detailed molecular studies were performed, revealing the presence of a normal 46,XY cell lineage in a vast majority of skin fibroblasts and a upid(AC)mat 46,XX cell lineage in nearly all blood cells. In addition, although the data are insufficient to draw a definitive conclusion. Horike et $a l^{3}$ have also identified 46,XX peripheral blood cells with possible upd(AC)mat in a phenotypically male patient through methylation analyses for plural differentially methylated regions (DMRs) in 11 patients with Silver-Russell syndrome (SRS)-like phenotype. This patient was found to have a normal 46,XY cell lineage and a triploid 69,XXY cell lineage in skin fibroblasts.

However, such patients with a upd(AC)mat cell lineage remain extremely rare, and there is no report describing a human with such a cell lineage in the absence of a normal cell lineage. Here, we report a female patient with a upid(AC)mat 46,XX cell lineage and a non-upd 45,X cell lineage who was identified through genetic screenings of 103 patients with SRS-like phenotype.

\section{MATERIALS AND METHODS Case report}

This Japanese female patient was conceived naturally and born at 40 weeks of gestation by a normal vaginal delivery. At birth, her length was $44.0 \mathrm{~cm}(-3.1 \mathrm{SD})$, her weight $2.1 \mathrm{~kg}(-2.9 \mathrm{SD})$ and her occipitofrontal head circumference (OFC) $30.5 \mathrm{~cm}(-2.3 \mathrm{SD})$. The parents and the younger brother were clinically normal (the father died from a traffic accident).

At 2 years of age, she was referred to us because of growth failure. Her height was $77.7 \mathrm{~cm}(-2.5$ SD), her weight $8.45 \mathrm{~kg}(-2.6 \mathrm{SD})$ and her OFC $43.5 \mathrm{~cm}$ ( $-2.5 \mathrm{SD})$. Physical examination revealed several SRS-like somatic features such as triangular face, right hemihypoplasia and bilateral fifth finger clinodactyly. She also had developmental retardation, with a developmental quotient of 56. Endocrine studies for short stature were normal as were radiological studies. Cytogenetic analysis using lymphocytes indicated a low-grade mosaic Turner syndrome (TS) karyotype, 45,X[3]/46,XX[47]. Thus, a screening of TS phenotype ${ }^{4}$ was performed, detecting horseshoe kidney but no body surface features or cardiovascular lesion. Chromosome analysis was repeated at 6 and 32 years of age using lymphocytes, revealing a 45,X[8]/46,XX[92] karyotype and a 45,X[12]/46,XX[88] karyotype, respectively. On the last examination at 34 years of age, her height was $125.0 \mathrm{~cm}$ (-6.2 SD), her weight $37.5 \mathrm{~kg}(-2.0 \mathrm{SD})$ and her OFC $51.2 \mathrm{~cm}(-2.8 \mathrm{SD})$. She was engaged in a simple work and was able to get on her daily life for herself.

\section{Sample preparation}

This study was approved by the Institutional Review Board Committees at National Center for Child health and Development. After obtaining written informed consent, genomic DNA was extracted from leukocytes of the patient, the mother and the brother and from salivary cells, which comprise $\sim 40 \%$ of buccal epithelial cells and $\sim 60 \%$ of leukocytes, ${ }^{5}$ of the patient. Lymphocyte metaphase spreads and leukocyte RNA were also 
obtained from the patient. Leukocytes of healthy adults and patients with imprinting disorders were utilised for controls.

\section{Primers and probes}

The primers utilised in this study are summarised in supplementary methods and supplementary tables $1-3$.

\section{DMR analyses}

We first performed bio-combined bisulfite restriction analysis (COBRA) ${ }^{6}$ and bisulfite sequencing of the H19-DMR (A) on chromosome $11 \mathrm{p} 15.5$ by the previously described methods ${ }^{7}$ and methylation-sensitive PCR analysis of the MEST-DMR (A) on chromosome 7q32.2 by the previously described methods ${ }^{8}$ with minor modifications (the methylated and unmethylated allelespecific primers were designed to yield PCR products of different sizes, and the PCR products were visualised on the 2100 Bioanalyzer (Agilent, Santa Clara, California, USA)). This was because hypomethylation (epimutation) of the normally methylated H19-DMR of paternal origin and maternal uniparental disomy 7 are known to account for $35-65 \%$ and $5-10 \%$ of SRS patients, respectively. ${ }^{9} 10$ In addition, fluorescence in situ hybridisation (FISH) analysis was performed with a $\sim 84-\mathrm{kb}$ RP5-998N23 probe containing the H19-DMR (BACPAC Resources Center, Oakland, California, USA). We also examined multiple other DMRs by bio-COBRA. The ratio of methylated clones (the methylation index) was calculated using peak heights of digested and undigested fragments on the 2100 Bioanalyzer using 2100 expert software.

\section{Genome-wide microsatellite analysis}

Microsatellite analysis was performed for 96 autosomal loci and $30 \mathrm{X}$ chromosomal loci. The segment encompassing each locus was PCR-amplified, and the PCR product size was determined on the ABI PRISM 310 autosequencer using GeneScan software (Applied Biosystems, Foster City, California, USA).

\section{PCR analysis for $\mathbf{Y}$ chromosomal loci}

Standard PCR was performed for six Y chromosomal loci. The PCR products were electrophoresed using the 2100 Bioanalyzer.

\section{Expression analysis}

Quantitative real-time reverse transcriptase PCR analysis was performed for three paternally expressed genes (IGF2, SNRPN and ZAC1) and four maternally expressed genes (H19, MEG3, PHLDA2 and CDKN1C) that are known to be variably (usually weakly) expressed in leukocytes (UniGene, http://www.ncbi. nlm.nih.gov/sites/entrez?db=unigene), using an ABI Prism 7000 Sequence Detection System (Applied Biosystems). TBP and GAPDH were utilised as internal controls.

\section{RESULTS \\ DMR analyses}

In leukocytes, the bio-COBRA indicated severely hypomethylated H19-DMR, and bisulfite sequencing combined with rs2251375 SNP typing for 30 clones revealed maternal origin of 29 hypomethylated clones and non-maternal (paternal) origin of a single methylated clone in this patient (figure 1A). Thus, the marked hypomethylation of the H19-DMR was caused by predominance of maternally derived clones rather than hypomethylation of the H19-DMR of paternal origin. FISH analysis for 100 lymphocyte metaphase spreads excluded an apparent deletion of the paternally derived H19-DMR or duplication of the maternally derived H19-DMR (Supplementary figure 1).
Methylation-sensitive PCR amplification for the MEST-DMR delineated a major peak for the methylated allele and a minor peak for the unmethylated allele (figure $1 \mathrm{~B}$ ). This also indicated the predominance of maternally derived clones and the coexistence of a minor portion of paternally derived clones. Furthermore, autosomal DMRs invariably exhibited markedly abnormal methylation patterns consistent with predominance of maternally inherited DMRs, whereas the methylation index of the XIST-DMR on the X chromosome remained within the female reference range (figure $1 \mathrm{C}$ ). The abnormal methylation patterns were less obvious in salivary cells (thus, in buccal epithelial cells) than in leukocytes, except for the methylation index for the XIST-DMR that mildly exceeded the female reference range (figure $1 \mathrm{~A}-\mathrm{C}$ ).

\section{Microsatellite analysis}

Major peaks consistent with maternal uniparental isodisomy and minor peaks of non-maternal (paternal) origin were identified for at least one locus on each autosome, with the minor peaks of non-maternal origin being more obvious in salivary cells than in leukocytes (figure 1D and supplementary table 4). Furthermore, the frequency of the upid(AC)mat cells was calculated as $84 \%$ in leukocytes, $56 \%$ in salivary cells and $18 \%$ in epithelial buccal cells, using the area under curves for the maternally and the non-maternally inherited peaks (supplementary note). Such minor peaks of non-maternal origin were not detected for all the $30 \mathrm{X}$ chromosomal loci examined.

\section{PCR analysis for $\mathbf{Y}$ chromosomal loci}

PCR amplification failed to detect any trace of Y chromosomespecific bands in leukocytes and salivary cells (Supplementary figure 2).

\section{Expression analysis}

Expression analysis using control leukocytes indicated that, of the seven examined genes, SNRPN expression alone was strong enough to allow for a precise assessment (Supplementary figure 3). SNRPN expression was extremely low in this patient (figure 1E).

\section{DISCUSSION}

These results imply that this patient had a upid(AC)mat 46,XX cell lineage and a non-upd 45,X cell lineage. Indeed, methylation patterns of the XIST-DMR is explained by assuming that the two $X$ chromosomes in the upid(AC)mat cells undergo random $\mathrm{X}$-inactivation and that $45, \mathrm{X}$ cells with the methylated XISTDMR on a single active $\mathrm{X}$ chromosome $\mathrm{e}^{11}$ are relatively prevalent in buccal epithelial cells. Furthermore, lack of non-maternally derived minor peaks for microsatellite loci on the $\mathrm{X}$ chromosome is explained by assuming that the two $\mathrm{X}$ chromosomes in the upid(AC)mat cells and the single X chromosome in the 45,X cells are derived from a common $\mathrm{X}$ chromosome of maternal origin, with no paternally derived sex chromosome. It is likely, therefore, that a parthenogenetic activation took place around the time of fertilisation of a sperm missing a sex chromosome, resulting in the generation of the 46,XX cell lineage with upid (AC)mat by endoreplication (the replication of DNA without the subsequent completion of mitosis) of one blastomere containing a female pronucleus and the 45,X cell lineage with biparentally derived autosomes and a maternally derived X chromosome by union of male and female pronuclei (figure 2), although it is also possible that a paternally derived sex chromosome was present in the sperm but was lost from the normal 
Figure 1 Representative molecular results. Pat, paternally derived allele; Mat, maternally derived allele;

$\mathrm{P}$, patient; $\mathrm{M}$, mother; $\mathrm{B}$, brother; $L$, leukocytes; and $S$, salivary cells. Filled and open circles in A and B represent methylated and unmethylated cytosine residues at the $\mathrm{CpG}$ dinucleotides, respectively. A Methylation patterns of the H19-DMR (A) harbouring $23 \mathrm{CpG}$ dinucleotides and the T/G SNP (rs2251375) (a grey box). The PCR products are digested with $B s a \mathrm{BI}$ when the cytosine at the sixth CpG dinucleotide (highlighted in yellow) is methylated and with $\mathrm{Mwol}$ when the two cytosines at the ninth and the 11th $\mathrm{CpG}$ dinucleotides (highlighted in orange) are methylated. For the bioCOBRA data, the black histograms represent the distribution of methylation indices (\%) in 50 control participants, and $L$ and $S$ denote the methylation indices for leukocytes and salivary cells of this patient, respectively. For the bisulfite sequencing data, each line indicates a single clone. B. Methylated and unmethylated allele-specific PCR analysis for the MEST-DMR (A). In a control participant, the PCR products for methylated and unmethylated alleles are delineated, and the unequal amplification is consistent with a short product being more easily amplified than a long product. In a previously reported patient with upd(7)mat, ${ }^{8}$ the methylated allele only is amplified. In this patient, major peaks for the methylated allele and minor peaks for the unmethylated allele (red asterisks) are detected. C. Methylation patterns for the 18 DMRs examined. The DMRs highlighted in blue and pink are methylated after paternal and maternal transmissions, respectively. The black vertical bars indicate the reference data (maximum-minimum) in 20 normal control participants, using leukocyte genomic DNA (for the XIST-DMR, 16 female data are shown).

D. Representative microsatellite analysis. Minor peaks (red asterisks) have been identified for D7S1824 and D11S904 but not for DXS986 of the patient. Since the peaks for D7S1824 and D11S904 are absent in the mother and clearly present in the brother, they are assessed to be of paternal origin. E. Relative expression level (mean \pm SD) of SNRPN on chromosome 15. The data have been normalised against TBP. SRS, an SRS patient with an epimutation (hypomethylation) of the H19-DMR; BWS1, a BWS patient with an epimutation (hypermethylation) of the H19-DMR; BWS2, a BWS patient with upd(11)pat; PWS1, a PWS patient with upd(15)mat; PWS2, a PWS patient with an epimutation (hypermethylation) of the SNRPN-DMR; AS1, an Angelman syndrome (AS) patient with upd(15)pat; and AS2, an AS patient with an epimutation (hypomethylation) of the SNRPN-DMR.

cell lineage at the very early developmental stage. Hence, in a strict sense, this patient is neither a chimera resulting from the fusion of two different zygotes nor a mosaic caused by a mitotic error of a single zygote. In this regard, a triploid cell stage is assumed in the generation of a upid(AC)mat cell lineage, and such triploid cells may have been detected in skin fibroblasts of the patient reported by Horike et al. ${ }^{3}$

The upid(AC)mat cells accounted for the majority of leukocytes even in adulthood of this patient, despite global negative selective pressure. $^{1213}$ This phenomenon, though intriguing, would not be unexpected in human studies because leukocytes are usually utilised for genetic analyses. Rather, if the upid(AC)mat cells were barely present in leukocytes, they would not have been detected. It is likely, therefore, that upid(AC)mat cells have occupied a relatively large portion of the definitive haematopoietic tissues primarily as a stochastic event. Furthermore, parthenogenetic chimera mouse studies have revealed that parthenogenetic cells are found at a relatively high frequency in some tissues/organs including blood and are barely identified in other tissues/organs such as skeletal muscle and liver. ${ }^{13}$ Such a possible tissue-specific selection in favour of the preservation of parthenogenetic cells in the definitive haematopoietic tissues may also be relevant to the predominance of the upid(AC)mat cells in leukocytes. In addition, a reduced growth potential of $45, \mathrm{X}$ cells ${ }^{14}$ may also have contributed to the skewed ratio of the two cell lineages.
Clinical features of this patient would be determined by several factors. They include: (1) the ratio of two cell lineages in various tissues/organs, (2) the number of imprinted regions or DMRs relevant to the development of specific imprinting disorders (eg, plural regions/DMRs on chromosomes 7 and 11 for SRS ${ }^{9} 10$ and a single region/DMR on chromosome 15 for Prader-Willi syndrome (PWS)), ${ }^{15}$ (3) the degree of clinical effects of dysregulated imprinted regions/DMRs (an (epi)dominant effect has been

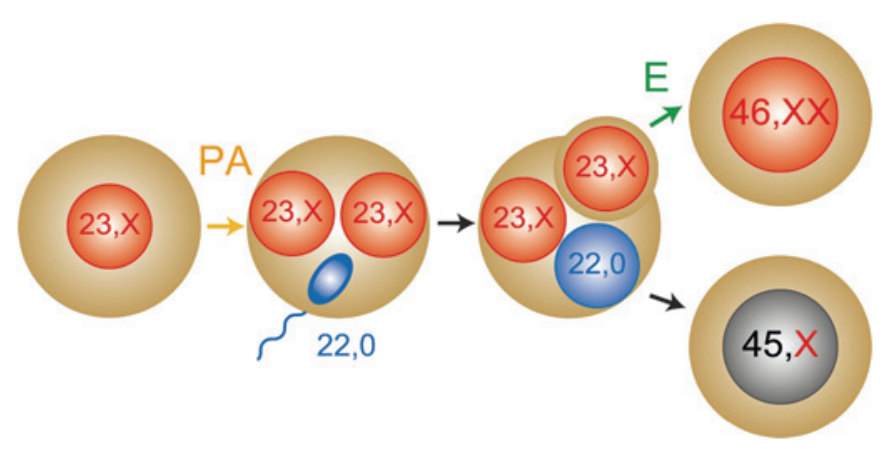

Figure 2 Schematic representation of the generation of the upid(AC) mat $46, X X$ cell lineage and the non-upd $45, X$ cell lineage. Polar bodies are not shown. $P A$, parthenogenetic activation; and $E$, endoreplication of one blastomere containing a female pronucleus. 
assumed for the $11 \mathrm{p} 15.5$ imprinted regions including the IGF2-H19 domain on the basis of SRS or Beckwith-Wiedemann syndrome (BWS) phenotype in patients with multilocus hypomethylation ${ }^{16}$ and BWS-like phenotype in patients with a upid (AC)pat cell lineage, ${ }^{17}$ a mirror image of a upid(AC)mat cell lineage), (4) expression levels of imprinted genes in upid(AC)mat cells (although SNRPN expression of this patient was consistent with upid(AC)mat cells being predominant in leukocytes, complicated expression patterns have been identified for several imprinted genes in androgenetic and parthenogenetic fetal mice, probably because of perturbed cis- and trans-acting regulatory mechanisms) ${ }^{18}$ and (5) unmasking of possible maternally inherited recessive mutation(s) in upid(AC)mat cells. ${ }^{19}$ Collectively, it appears that the extent of overall (epi)genetic aberrations exceeded the threshold level for the development of SRS phenotype and horseshoe kidney characteristic of $\mathrm{TS}^{4}$ but remained below the threshold level for the occurrence of other imprinting disorders or recessive Mendelian disorders.

In summary, we identified a upid(AC)mat 46,XX cell lineage in a woman with an SRS-like phenotype and a 45,X cell lineage accompanied by autosomal haploid sets of biparental origin. This report will facilitate further identification of patients with a upid(AC)mat cell lineage and better clarification of the clinical phenotypes in such patients.

Acknowledgements We thank the patient and her family members for their participation in this study. We also thank Dr. Toshiro Nagai for providing us with blood samples of patients with Prader-Willi syndrome.

Funding This work was supported by grants from the Ministry of Health, Labor, and Welfare and from the Ministry of Education, Science, Sports and Culture.

Competing interests None.

Patient consent Obtained.

Ethics approval This study was conducted with the approval of the Institutional Review Board Committees at National Center for Child health and Development.

Contributors Drs Kazuki Yamazawa (first author) and Kazuhiko Nakabayashi (second author) contributed equally to this work.

Provenance and peer review Not commissioned; externally peer reviewed.

\section{REFERENCES}

1. McGrath J, Solter D. Completion of mouse embryogenesis requires both the maternal and paternal genomes. Cell 1984;37:179-83.

2. Strain L, Warner JP, Johnston T, Bonthron DT. A human parthenogenetic chimaera. Nat Genet 1995;11:164-9.
3. Horike S, Ferreira JC, Meguro-Horike M, Choufani S, Smith AC, Shuman C, Meschino W, Chitayat D, Zackai E, Scherer SW, Weksberg R. Screening of DNA methylation at the $\mathrm{H} 19$ promoter or the distal region of its ICR1 ensures efficient detection of chromosome 11p15 epimutations in Russell-Silver syndrome. Am J Med Genet Part A 2009;149A:2415-23.

4. Styne D, Grumbach M. Puberty: ontogeny, neuroendocrinology, physiology, and disorders. In: Kronenberg H, Melmed M, Polonsky K, Larsen P, eds. Williams textbook of endocrinology, 11th edn. Philadelphia: Saunders 2008:969-1166.

5. Thiede C, Prange-Krex G, Freiberg-Richter J, Bornhauser M, Ehninger G. Buccal swabs but not mouthwash samples can be used to obtain pretransplant DNA fingerprints from recipients of allogeneic bone marrow transplants. Bone Marrow Transplant 2000;25:575-7.

6. Brena RM, Auer H, Kornacker K, Hackanson B, Raval A, Byrd JC, Plass C. Accurate quantification of DNA methylation using combined bisulfite restriction analysis coupled with the Agilent 2100 Bioanalyzer platform. Nucleic Acids Res 2006; 34:e17

7. Yamazawa K, Kagami M, Nagai T, Kondoh T, Onigata K, Maeyama K, Hasegawa T, Hasegawa Y, Yamazaki T, Mizuno S, Miyoshi Y, Miyagawa S, Horikawa R, Matsuoka K, Ogata T. Molecular and clinical findings and their correlations in Silver-Russell syndrome: implications for a positive role of IGF2 in growth determination and differential imprinting regulation of the IGF2-H19 domain in bodies and placentas. J Mol Med 2008;86:1171-81.

8. Yamazawa K, Kagami M, Ogawa M, Horikawa R, Ogata T. Placental hypoplasia in maternal uniparental disomy for chromosome 7. Am J Med Genet Part A 2008:146A:514-16.

9. Abu-Amero S, Monk D, Frost J, Preece M, Stanier P, Moore GE. The genetic aetiology of Silver-Russell syndrome. J Med Genet 2008;45:193-9.

10. Eggermann T, Eggermann K, Schonherr N. Growth retardation versus overgrowth: Silver-Russell syndrome is genetically opposite to Beckwith-Wiedemann syndrome. Trends Genet 2008;24:195-204.

11. Goto T, Monk M. Regulation of X-chromosome inactivation in development in mice and humans. Microbiol Mol Biol Rev 1998;62:362-78.

12. Nagy A, Sass M, Markkula M. Systematic non-uniform distribution of parthenogenetic cells in adult mouse chimaeras. Development 1989:106:321-4.

13. Fundele R, Norris ML, Barton SC, Reik W, Surani MA. Systematic elimination of parthenogenetic cells in mouse chimeras. Development 1989;106:29-35.

14. Verp MS, Rosinsky B, Le Beau MM, Martin AO, Kaplan R, Wallemark CB, Otano L, Simpson JL. Growth disadvantage of $45, X$ and $46, X$, del(X)(p11) fibroblasts. Clin Genet 1988; 33:277-85.

15. Horsthemke B, Wagstaff J. Mechanisms of imprinting of the Prader-Willi/ Angelman region. Am J Med Genet A 2008;146A:2041-52.

16. Azzi S, Rossignol S, Steunou V, Sas T, Thibaud N, Danton F, Le Jule M, Heinrichs C Cabrol S, Gicquel C, Le Bouc Y, Netchine I. Multilocus methylation analysis in a large cohort of 11p15-related foetal growth disorders (Russell Silver and Beckwith Wiedemann syndromes) reveals simultaneous loss of methylation at paternal and maternal imprinted loci. Hum Mol Genet 2009;18:4724-33.

17. Wilson M, Peters G, Bennetts B, McGillivray G, Wu ZH, Poon C, Algar E. The clinica phenotype of mosaicism for genome-wide paternal uniparental disomy: two new reports. Am J Med Genet Part A 2008;146A:137-48.

18. Ogawa H, Wu Q, Komiyama J, Obata Y, Kono T. Disruption of parentalspecific expression of imprinted genes in uniparental fetuses. FEBS Lett 2006;580:5377-84.

19. Engel E. A fascination with chromosome rescue in uniparental disomy: Mendelian recessive outlaws and imprinting copyrights infringements. Eur J Hum Genet 2006:14:1158-69. 